\title{
Cardiac arrest survival post-resuscitation in-hospital (CASPRI) - a tool to predict neurological outcome after ROSC
}

\section{Bofu Liu}

Sichuan University West China Hospital

Yarong He

Sichuan University West China Hospital

Peng Jiang

Sichuan University West China Hospital

Jiachen Sun

Sichuan University West China Hospital

Tianyong Han

Sichuan University West China Hospital

\section{Qin Li}

Sichuan University West China Hospital

\section{Ruixin Li}

Sichuan University West China Hospital

Yu Cao ( $\nabla$ yuyuer@126.com )

https://orcid.org/0000-0003-4715-7331

\section{Research}

Keywords: CASRPI score; in-hospital cardiac arrest; return of spontaneous circulation; short-term neurological prognosis

Posted Date: March 17th, 2020

DOl: https://doi.org/10.21203/rs.3.rs-17394/v1

License: (c) (1) This work is licensed under a Creative Commons Attribution 4.0 International License. Read Full License 


\section{RESEARCH}

\section{Cardiac arrest survival post-resuscitation in-hospital (CASPRI)—a tool to predict neurological outcome after ROSC}

Bo-fu Liu ${ }^{1,2,3 \dagger}$, Ya-rong He $\mathrm{He}^{1,2,3 \dagger}$, Peng Jiang ${ }^{1,2,3}$, Jia-chen Sun ${ }^{4}$, Tian-yong Han ${ }^{1,4}$, Qin Li ${ }^{1,4}$, Rui-xin $\mathrm{Li}^{1,4}$ and $\mathrm{Yu} \mathrm{Cao}^{1,2,3^{*}}$

\footnotetext{
${ }^{*}$ Correspondence: yuyuer@126.com

${ }^{1}$ Department of Emergency

Medicine, West China Hospital,

Sichuan University, Guoxue Road

37, 610041 Chengdu, China

Full list of author information is

available at the end of the article

${ }^{\dagger}$ Equal contributor
}

\begin{abstract}
Objectives: To develop a cardiac arrest survival post-resuscitation in-hospital (CASPRI) scoring system evaluating the prognosis of neurological function in ROSC patients.
\end{abstract}

Methods: This single-center, retrospective study reviewed the eligible patients admitted to the emergency department of West China Hospital of Sichuan University who received cardiopulmonary resuscitation and restored spontaneous circulation from January 1, 2014 00:00 to December 31, 2017 23:59. Clinical histories, blood test, biochemistry profile, coagulation indexes and other laboratory tests during emergency department visit were collected. The MEWS, sOHCA, APACHE II, and the highest SOFA scores were calculated during the period between emergency room admission and ROSC. The clinical data of ROSC patients in the test group were analyzed by univariate and multivariate logistic analysis. The possible risk factors related to the unfavorable prognosis of 90-day neurological function were screened and CASPRI score was constructed. The efficacy of CASPRI score on evaluating the neurological function of ROSC patients was analyzed by ROC curve and proved in the validation group.

Main results: 503 patients were included in the test group. After correcting potential confounding factors, multivariate logistic regression analysis showed that TBIL, ALB at admission of emergency department, Lac at ROSC, resuscitation time, non-shockable rhythms were independent risk factors for poor neurological prognosis of ROSC patients $(p<0.05)$. ROC curve showed that the CASPRI score was superior to the APACHE II score, SOFA score, MEWS score and $\mathrm{SOHCA}$ score, and the difference was statistically significant $(\mathrm{p}<0.05)$. In the validation group with 256 patients included, the incidence of poor neurological prognosis in high-risk, intermediate-risk, and low-risk groups based on CASPRI score were $97.89 \%, 85.59 \%$, and $58.33 \%$, respectively, and the difference was statistically significant $(p<0.001)$. CASPRI score was superior to the SOFA score and MEWS score, the difference was statistically significant $(p<0.05)$.

Conclusions: CASPRI score is an effective tool for the early evaluation of the neurological prognosis of ROSC patients. Its efficacy exceeds the MEWS and SOFA scoring systems currently used in clinical practice.

Trial registration: This study was approved by China Ethics Committee of Registering Clinical Trials (chiECRT-20180032).

Keywords: CASRPI score; in-hospital cardiac arrest; return of spontaneous circulation; short-term neurological prognosis 


\section{Introduction}

With the improvement of cardiopulmonary resuscitation (CPR), the resuscitation success rate of the in-hospital cardiac arrest (IHCA) patients has been increased. However, after return of spontaneous circulation (ROSC), the survival rate of discharged patients and their neurological prognosis remain unsatisfactory $[1,2,3]$. A multi-center study in China has shown that only $6.4 \%$ of patients with IHCA had good recovery of neurological functions [4]. Hence, it is an urgent need for indicators or tools that can be used to accurately and quickly assess the neurological prognosis of ROSC patients. Existing studies have pointed that whether the witnesses started CPR, "no blood flow" and low perfusion time, the initial rhythm of cardiac arrest, laboratory examinations during hospital visit, and other indicators could predict the neurological prognosis of ROSC patients to some extent $[5,6]$, but only to a limited degree [7]. Studies have also suggested that simplified out-of-hospital cardiac arrest (sOHCA) [8], sequential organ failure assessment (SOFA) [9], acute physiology and chronic health evaluation II (APACHE II) [10], and modified early warning score (MEWS) [11] can be adopted to evaluate the neurological prognosis of ROSC patients. Nevertheless, these scoring systems share the disadvantages, such as complicated calculation, tedious indicators, and requirement of continuous dynamic evaluation.

This study intends to analyze the clinical data of ROSC patients through a retrospective cohort study, screen independent risk factors that affect their short-term neurological prognosis, and create a new scoring system to enable a rapid, simple and accurate assessment of their neurological prognosis. The purpose is to provide a basis for clinicians to make medical decisions and communicate with patient's families.

\section{Methods}

\section{Patients}

The clinical management charts of eligible patients admitted to the emergency department of West China Hospital of Sichuan University who received cardiopulmonary resuscitation and resumed spontaneous circulation through cardiopulmonary resuscitation from January 1, 2014 00:00 to June 30, 2018 23:59 were reviewed (Figure 1). Inclusion criteria was IHCA patients who underwent CPR and ROSC. Exclusion criteria included age less than 18, CPR performed outside the hospital at the same visit, abandoned treatment by families, pregnancy, previous neurological deficits, traumatic brain injury or primary central nervous system diseases. The ROSC patients from January 1, 2014 00:00 to December 31, 2016 23:59 was set as test group, other ROSC patients were assigned to verification group.

\section{Sample size estimation}

Assuming that the AUC of the test group reaches at least 0.70, the test level $\alpha=$ 0.05 , the test efficiency is $1-\beta=0.95$, and the estimated missed follow-up rate was $10 \%$. The calculated minimum sample size is 330 cases. Assuming that the AUC of the verification group reaches at least 0.75 , the test level $\alpha=0.05$, the test efficiency $1-\beta=0.9$, and the estimated missed follow-up rate is $10 \%$. The minimum sample size of the verification group is 170 cases. The total sample size requires at least 500 cases. These calculations are based on Medcalc software. 


\section{Measurements}

Clinical histories, vital signs, including temperature $(\mathrm{T})$, heart rate $(\mathrm{HR})$, respiration rate $(\mathrm{RR})$, oxygen saturation $\left(\mathrm{SpO}_{2}\right)$, systolic blood pressure ( $\left.\mathrm{SBP}\right)$, diastolic blood pressure (DBP). First series of laboratory tests during emergency room visit, red blood cell (RBC), hemoglobin (Hb), hematocrit (HCT), white blood cell (WBC), platelet (PLT), total bilirubin (TBIL), alanine aminotransferase (ALT), aspartate transaminase (AST), albumin (ALB), glucose (GLU), creatinine $(\mathrm{Cr})$, triglyceride (TG), cholesterol (CHOL), serum potassium $\left(K^{+}\right)$, serum sodium $\left(\mathrm{Na}^{+}\right)$, prothrombin time (PT), activated partial thromboplastin time (APTT), fibrinogen (FIB) and D-dimer. Lactic acid (Lac) during resuscitation, the resuscitation time, shockable or non-shockable rhythm. The modified early warning score (MEWS), simplified out-of-hospital cardiac arrest (sOHCA) score, acute physiology and chronic health evaluation II (APACHE II), and the highest sequential organ failure assessment (SOFA) score were calculated during the period between emergency room admission and ROSC. According to their cerebral performance category (CPC) score at day 90, patients included were divided into favorable prognosis group (CPC 1-2) or unfavorable prognosis group (CPC 3-5). The clinical data of ROSC patients in the test group were analyzed by univariate and multivariate logistic analysis. The possible risk factors related to the unfavorable prognosis of neurological function in 90-day were screened and CASPRI score was constructed.

\section{Follow-up and monitoring}

Hospitalized patients were followed up by HIS system and ward visits to observe the endpoints. Discharged patients were followed by telephone or outpatient visits. Before data collection, the research purpose and research route are consulted to the mentor and expert group to formulate a scientific and feasible research plan. Physicians who have been intern in our hospital for more than half a year are selected for data collection in this study. Uniform training before data collection, data collection in strict accordance with the inclusion and exclusion criteria, including viewing computer medical records, nursing records, paper medical records, and telephone follow-up of ROSC patients for 90-day Neurological conditions. The researcher is involved in the whole process of data recording, and the double-checking corrects the entered information errors in time.

\section{Statistics}

SPSS 21.0 and MedCalc 18.2.1 were used for data analysis. Measured data that conform to the normal distribution is expressed as mean \pm standard deviation, and comparison between the two groups was performed by independent-sample t-test; measured data that do not conform to the normal distribution is expressed as the median and interquartile range, and the rank-sum test was used for comparison between groups. Enumeration data are expressed by frequency and percentage, and the chi-square test or Fisher exact test were adopted.

A statistical method of CASPRI score was established in the test group. A univariate binary Logistic regression analysis was used to analyze the risk factors that may affect the prognosis of ROSC patients. The odds ratio (OR) and $95 \%$ confidence interval (CI) were calculated. Potential risk factors with $\mathrm{p}<0.05$ were selected for 
multivariate logistic regression analysis. Bilateral p-values $<0.05$ were considered to be independently related to poor prognosis in ROSC patients. The AUC was used to obtain the intercept value of the continuous variable. Continuous variables were converted to categorical variables according to the cutoff value, and then logistic regression analysis was performed to obtain $\beta$ regression coefficients for each independent risk factor. Then, a CASPRI score was created based on the least common divisor of the $\beta$ regression coefficients, and the score of each ROSC patient was calculated.

In the test group, ROC curve analysis was used to compare the efficacy of CASPRI score and other scoring systems to predict the poor prognosis of ROSC patients, and the efficacy of CASPRI score to evaluate the poor prognosis of ROSC patients with different disease types.

In the validation group, the CASPRI score was divided into three groups (low-risk group, intermediate-risk group, and high-risk group) according to the trisection of the incidence of poor prognosis, and the CASPRI score in evaluating the criticality of ROSC patients was verified. This study used two-sided p-values, $\mathrm{p}<0.05$ was statistically significant.

\section{Results}

Flow of patients

A total of 801 patients (Figure 1) with successful resuscitation in the emergency department were collected, 42 patients were lost to follow-up, with the loss of followup rate of $5.24 \%$, and 759 cases were eventually included. Patients included in the study were mostly male (64.43\%), the average age was $60.12 \pm 18.11$ years old. 97 patients $(12.78 \%)$ had good neurological outcomes on day 90 .

Comparison of clinical baseline between patients in the test group and the verification group

The difference between test group and validation group in general information (gender, age), vital signs (temperature, heart rate, respiratory rate, blood pressure, oxygen saturation), laboratory tests (blood routine, biochemistry profile, coagulation indexes), scale of disease types, and the incidence of endpoint events were not significant $(\mathrm{p}>0.05$; Table 1$)$.

\section{Construction of CASPRI model}

\section{Baseline data of patients in the test group}

A total of 503 patients were enrolled, with an average age of $60.11 \pm 18.62$ years old, including 323 males (64.21\%) and 180 females (35.79\%). The patients of the favorable prognosis group and the unfavorable prognosis group were $59(11.73 \%)$ and $444(88.27 \%)$, respectively. The difference of heart rate (HR), blood pressure (BP), respiratory rate $(\mathrm{RR})$, oxygen saturation $\left(\mathrm{SpO}_{2}\right)$, platelet count (PLT), activated partial thromboplastin time (APTT), D-dimer, lactic acid (Lac) during resuscitation, the resuscitation time, defibrillation rhythm, or cardiogenic causes between the two group were statistically significant ( $\mathrm{p}<0.05$; Table 2 ). MEWS, SOFA, APACHE II, and sOHCA scores in the poor prognosis group were significantly higher than those in the favorable prognosis group $(\mathrm{p}<0.05$; Table 2$)$. 
Independent risk factors for the poor neurological prognosis of ROSC patients Logistic regression analysis showed that $\mathrm{HR}, \mathrm{RR}, \mathrm{SpO}_{2}, \mathrm{PLT}$, total bilirubin (TBIL), ALB, PT and APTT at the admission of emergency department, Lac at ROSC, resuscitation time, non-shockable rhythms and non-cardiogenic causes showed significant correlations $(\mathrm{p}<0.05)$ with poor prognosis of ROSC patients.

After correcting potential confounding factors, multivariate logistic regression analysis showed that TBIL, ALB at the admission of emergency department, Lac at ROSC, resuscitation time, non-shockable rhythms were independent risk factors for poor prognosis of ROSC patients ( $\mathrm{p}<0.05$; Table 3 ). According to receiver operating characteristic (ROC) curve analysis (Table 4; Figure 2), the cutoff values of independent risk factors were TBIL $(23.4 \mu \mathrm{mol} / \mathrm{L})$, ALB $(34.5 \mathrm{~g} / \mathrm{L})$, Lac $(5.3 \mathrm{mmol} / \mathrm{L})$ and resuscitation time (9 $\mathrm{min})$.

Construction of CASPRI scoring model

According to the regression coefficients of multivariate logistic regression, the indexes assigned included: TBIL $(\leq 24.6 \mu \mathrm{mol} / \mathrm{L}$ score 0 points, $>24.6 \mu \mathrm{mol} / \mathrm{L}$ score 3 points), ALB ( $>34.5 \mathrm{~g} / \mathrm{L}$ score 0 points, $\leq 34.5 \mathrm{~g} / \mathrm{L}$ score 2 points $)$, Lac $(\leq 5.3 \mathrm{mmol} / \mathrm{L}$ score 0 points, $>5.3 \mathrm{mmol} / \mathrm{L}$ score 2 points), resuscitation time ( $\leq 9$ minutes score 0 points, $>9$ minutes score 2 points), whether the heart rhythm is shockable ( 0 points for shockable rhythm, 2 points for non-shockable rhythm), cardiogenic causes (cardiogenic causes score 0 points, non-cardiac causes score 1 point) (Table 5).

The efficacy of CASPRI score on assessing the poor prognosis in ROSC patients in the test group

Multivariate logistic regression analysis found that CASPRI score was an independent risk factor for poor prognosis in ROSC patients. The area under the curve (AUC) of CASPRI score predicting poor prognosis of ROSC patients was 0.896 (95\% $\mathrm{CI}=0.851-0.941, \mathrm{p}=0.000$ ), with a sensitivity of $74.32 \%$ and specificity of $91.53 \%$, while the positive predictive value was $98.5 \%$ and negative predictive value was $32.1 \%$. The positive likelihood ratio was 8.77 , the negative likelihood ratio was 0.09 (Table 6). Compared with other scoring systems, the CASPRI score (AUC=0.896) was superior to the APACHE II score $(\mathrm{AUC}=0.752)$, SOFA score $(\mathrm{AUC}=815)$, MEWS score $(\mathrm{AUC}=0.599)$ and sOHCA score $(\mathrm{AUC}=0.829)$, and the difference was statistically significant $(\mathrm{p}<0.05$; Figure 3 ; Table 7$)$.

Verification of CASPRI score in evaluating the neurological prognosis of ROSC patients

Baseline characteristics of patients in the validation group

A total of 256 patients were enrolled, including 166 male patients (64.84\%), 96 female $(35.16 \%)$, and aged $60.26 \pm 17.19$ years old. The number of patients in the favorable prognosis group and the unfavorable prognosis group were 38 (14.84\%) and $218(85.15 \%)$, respectively.

Efficacy of the CASPRI score in evaluating the neurological prognosis of ROSC patients in validation group

The incidence of poor prognosis in patients with high-risk, intermediate-risk, and low-risk groups with CASPRI scores was $97.89 \%, 85.59 \%$, and $58.33 \%$, respectively, 
and the difference was statistically significant $(\mathrm{p}<0.001$; Figure 4$)$. In the validation group, the AUC of CASPRI score predicting prognosis of a 90-day neurological deficit in ROSC patients was 0.831 (95\% CI=0.780-0.875, $\mathrm{p}=0.000)$, with the sensitivity of $78.95 \%$ and the specificity of $72.94 \%$. The positive predictive value was $95.2 \%$, while the negative predictive value was $33.7 \%$, the positive likelihood ratio was 2.9 , and the negative likelihood ratio was 0.34 . Compared with other scores, the CASPRI score $(\mathrm{AUC}=0.831)$ was superior to the SOFA score $(\mathrm{AUC}=0.726)$ and MEWS score $(\mathrm{AUC}=0.693)$, the difference was statistically significant $(\mathrm{p}<0.05$; Figure 4 and 5 ; Table 8 and 9 ).

\section{Discussion}

Independent risk factors for the short-term neurological prognosis in ROSC patients

Elevation of serum bilirubin was an independent risk factor for poor prognosis of neurological function at day 90 in ROSC patients $(\mathrm{OR}=1.040,95 \% \mathrm{CI}=1.007-1.075$, $\mathrm{p}=0.017)$. Neurological dysfunction caused by serum bilirubin can be manifested as abnormalities in cognitive function, learning ability, motor coordination, and other aspects [12], while serum bilirubin that causes neurological dysfunction is mainly free bilirubin. Under normal conditions, low concentrations of endogenous bilirubin produced by brain tissue have protective effects on nerve cells. In various pathological states (e.g., after CPR), free bilirubin and non-free bilirubin enter the brain via the damaged blood-brain-barrier, reduce the activity of $\mathrm{Na}^{+}-\mathrm{K}^{+}$ATPase and prevent nerve cells from generating action potential [13]. This results in a decreased excitability toward stimulus and causes nerve cell damage, and thus with a poor neurological prognosis [14]. Ye et al. found a potential link between hyperbilirubinemia and auditory neuropathy [15]. Loftspring et al. demonstrated that unbound bilirubin increased cerebral edema and neutrophil activation in mice with cerebral hemorrhage [16]. Thus hyperbilirubinemia worsens the prognosis of neurological function. In addition, hyperbilirubinemia has also been associated with poor prognosis in critical patients. Gul et al. [17] measured total bilirubin in 1624 STEMI patients after PCI and found that the in-hospital in the high DBIL group was significantly higher than that in the low DBIL group ( $4 \%$ vs.1.5\%, $\mathrm{p}=0.003$ ), and multivariate analysis suggested a significant correlation between high DBIL levels and cardiovascular mortality in hospitalized patients (OR: 3.24, 95\%CI: 1.27-8.27, $\mathrm{p}=0.014$ ). Our results are consistent with the above studies. The prevalence of adverse neurological function in patients with hyperbilirubinemia before resuscitation is significantly higher than that in patients with hypobilirubinemia, which suggests more free bilirubin and non-free bilirubin enter brain tissue through the impaired blood-brain barrier, which causes nerve cell damage by reducing the excitability of nerve cells, induces brain edema, and leads to a poor prognosis of neural function. First serum albumin test level after admission to the emergency department was an independent risk factor for a 90-day poor neurological prognosis in ROSC patients. Albumin is an important plasma protein with various important functions such as substance transportation, maintenance of normal oncotic pressure, control of blood viscosity and platelet aggregation [18]. Leite et al. [19] has found that low serum albumin was associated with higher mortality - every $1 \mathrm{~g} / \mathrm{dL}$ increase in serum albumin at admission reduced the risk of death by $73 \%$ (OR: $0.27,95 \% \mathrm{CI}$ : 
0.14-0.51: $\mathrm{P}<0.001)$. Similarly, many other studies have confirmed that albumin is closely related to the prognosis of neurological function [20, 21]. Our study is consistent with the results mentioned above. It is speculated that the reason may be that the body's liver function is impaired in a critical state and a stress reaction characterized by high metabolism, which increases serum protein consumption and reduces synthesis; systemic capillary permeability increases, intravascular proteins are transferred to the interstitial space; and albumin is degraded to synthesize inflammatory factors, acute phase response proteins, resulting in a decrease in serum albumin, which leads to reduced effective circulating blood volume, microcirculation disorders, multiple organ dysfunction, defected immune system and increased chance of infection increase patient mortality [22].

Cardiopulmonary resuscitation time has been considered to be an important factor affecting the prognosis of cardiac arrest patients Saghafinia et al. [23] demonstrated that resuscitation time was significantly correlated with patient survival and discharge rate, and that the survival rate of patients with a resuscitation over 10 minutes decreased significantly. Gomes et al. [24] showed that the shorter the resuscitation time, the better the prognosis. A duration of less than 5 minutes is a favorable factor for the short-term prognosis, and the resuscitation time less than 15 minutes is an independent predictor of long-term survival. Arrigo et al. [2] reviewed 11 articles with 856 cardiac arrest patients receiving CPR and found that the survival rate and neurological prognosis were closely related to resuscitation time. Wang et al. [25] evaluated 16 studies with a total of $1162 \mathrm{CPR}$ patients and found that resuscitation time was an independent predictor of survival and poor neurological prognosis (CPC 3-5) in cardiac arrest patients. Our results support the above findings.

The neurological prognosis of patients with non-shockable rhythm during cardiac arrest was significantly worse than those shockable, and non-shockable rhythm was an independent risk factor for 90-day poor neurological prognosis in ROSC patients. There might be a pathophysiological explanation for this observation: in asystole or PEA, there is neither electrical nor mechanical activity in the heart; whereas in $\mathrm{VT} / \mathrm{VF}$, the arrested heart had certain levels of electrical activity that failed to translate to mechanical pumping [26].In addition, VT and VF have been considered "good" cardiac arrest rhythms [27], they can be quickly and successfully treated through defibrillation [28]. Our results showed that the rate of shockable heart rhythm was so low at cardiac arrest, accounting for only about $8.8 \%$, which is quite different from most other reports $[28,29,30]$. This difference can be due to the situation that most critically ill patients in our hospital are referred from lower-level hospitals, they might catch "not good" cardiac arrest rhythms because of prearrest illness severity and comorbidities.

The patients with non-cardiogenic etiology had a worse neurological prognosis. Cardiogenic diseases include unstable angina, acute coronary syndromes, and diseases such as heart failure. Cooper et al. [31] and Kaki et al. [32] analyzed 808 and 4631, respectively, IHCA patients and both found that cardiogenic CA patients had higher discharge survival rates than non-cardiogenic CA patients. Our results are consistent with them. When various reasons cause acute disturbance of coronary circulation, the myocardium may suffer from acute ischemia, hypoxic injury or even 
necrosis. The damaged cardiac muscle fibers may have different refractory periods from normal ones, and it is easy to form reentrant excitement. The initial rhythm of cardiogenic cardiac arrest is mostly $\mathrm{VF} / \mathrm{VT}$, then defibrillation is given after timely detection when the homeostasis has not deviate significantly, and so the prognosis is good. There are various disturbances on the homeostasis when non-cardiogenic cardiac arrest occurs, even supported with resuscitation and drugs, the causes of cardiac arrest cannot be removed in a short time, so it leads to a prolonged resuscitation time, prolonged ischemia and hypoxia in important organs such as the brain, and ultimately leads to poor neurological prognosis [33].

The level of plasma lactic acid during ROSC was higher in the poor prognosis group than the good prognosis group, and it was an independent factor for the 90-day poor neurological prognosis of ROSC patients. Lactic acid reflects the perfusion of tissues and organs. A retrospective study of 236 cases of cardiac arrest by Dell'Anna et al. [34] found that the first lactic acid concentration after ROSC was an independent risk factor for patients with poor neurological prognosis at 3 months. Zhou et al. [35] conducted a meta-analysis of 23 studies involving a total of 6720 cardiac arrest patients and showed that patients with good neurological prognosis had lower plasma lactate concentrations than those with poor prognosis at admission, $12 \mathrm{~h}$, $24 \mathrm{~h}$, and $48 \mathrm{~h}$ after resuscitation. During cardiac arrest and CPR, the anaerobic glycolytic pathway is activated to cause lactic acid accumulation and microcirculation disorders, which further aggravate organ and tissue ischemia and hypoxia, thereby causing organ dysfunction, and leading to poor neurological prognosis.

Lower plasma platelet level and high levels of PT, APTT, and D-dimer at admission are ominous for ROSC patients. Khurana et al. [36] found that low platelet count and its progressive decline at admission indicated the severity of critically ill patients and were directly related to prognosis and mortality in ICU patients. Akca et al. [37] studied 1,440 patients admitted to the ICU and showed that $30 \%$ of patients had a reduced platelet count at admission $\left(\mathrm{PLT}<150 \times 10^{9} / \mathrm{L}\right)$, and the survivors had higher platelet counts. During the inflammatory period before CPR, reduced production or increased depletion of platelets leads to thrombocytopenia, which can cause microvascular thrombosis and multiple organ dysfunction [21]. The dysregulated platelets interact with vascular endothelium, lead to leakage of inflammatory factors and further damage brain tissue [38]. Buchtele et al. [39] studies 388 patients with out-of-hospital cardiac arrest that $8 \%$ had DIC upon admission, and the D-dimer level in the 30-Day death group after admission was significantly higher than those who survived. Most critically ill patients, especially those with impaired liver function, have limited coagulation factors synthesis and decreased ability to clear metabolic waste during coagulation. Therefore, they are prone to thrombosis, bleeding, and in severe cases, DIC.

\section{Efficacy of different scoring system in predicting 90-day neurological prognosis in ROSC patients}

In this study, APACHE II, SOFA, MEWS, sOHCA, and CASPRI scores predicted the 90-day neurological prognosis of ROSC patients, nevertheless, CASPRI score had a greater AUC than other scoring systems in both the test group and the validation group. 
Our data suggests that APACHE II and SOFA scores effectively predicted the neurological prognosis of ROSC patients, which is consistent with previews studies on APACHE II [40, 41] and SOFA [42, 43, 44]. However, in this study, their predictive performance was not as good as the CASPRI score. The scores of severe organ dysfunction or immune impairment in the APACHE II score do not have clear standards and subdivisions, and are subject to more subjective factors. The variables such as body temperature, heart rate, and respiratory rate included in APACHE II are not independent risk factors that affect the prognosis of patients. In SOFA score, PLT and TBIL weighted more than which in the CASPRI score. And it has showed that SOFA has limited accuracy in prognostic evaluation of critically ill patients with single system severe dysfunction [44]. Moreover, there are many indicators to collect for the APACHE II score, and blood gas analysis results are difficult to collect in some hospitals. SOFA score requires continuous evaluation and complex calculation. Therefore, APACHE II and SOFA scores are not suitable for the needs of simple and rapid assessment in emergency department.

DeVoe et al. [45] found that the MEWS score at admission was a predictor of death in IHCA patients. However, in agreement with Meester et al. [46], our study showed poor sensitivity and specific of MEWS score, which had a significantly less AUC than the CASPRI score. Although the indexes such as RR, HR, and SBP at admission were different between the good prognosis group and the poor prognosis group, they were not caused by the independent risk factors.

We found that sOHCA had a moderate predictive effect in the prognosis of ROSC patients $(\mathrm{AUC}=0.811, \mathrm{P}<0.001,95 \% \mathrm{CI}=0.78-0.84)$, which was slightly lower than the CASPRI score, but this difference is not statistically significant. Both scores are specific for prognosis of ROSC patients, which depend on independent risk factors that affect the prognosis, and contain non-shockable rhythm and resuscitation time. CARPRI score contains more independent risk factors than sOHCA score, and is more comprehensive. Although the indexes in sOHCA are easy to obtain without a dynamic monitoring, the calculation is still complicated and not suitable for emergency use.

CASPRI score consists of independent risk factors affecting the prognosis of ROSC patients - resuscitation time, non-shockable rhythm, non-cardiogenic etiologies, platelets, white blood cells, and total bilirubin. The HL test showed that the CASPRI score has a good information extraction capabilities. We found it has a moderate predictive performance on patients' prognosis, which performed better than other scores. Because it's simple and quick to calculate, CASPRI score can help clinicians make medical decisions and better communicate with patients' families. In addition, CASPRI score is able to separate ROSC patients into low, medium and high risk groups, thus can guide the risk stratification of patients.

\section{Conclusions}

The CASPRI score can better predict the short-term neurological prognosis of ROSC patients, and its evaluation efficacy is better than the existing SOFA and MEWS scores. It can well distinguish the criticality of ROSC patients, and it is related to the incidence of short-term neurological prognosis. 
Availability of data and materials

The datasets used and/or analysed during the current study are available from the corresponding author on reasonable request.

\section{Abbreviations}

ALT: Alanine aminotransferase; ALB: Albumin; AST: Aspartate transaminase; APACHE II: Acute physiology and chronic health evaluation II; APTT: Activated partial thromboplastin time; ASY: Asystole; AUC: Area under the curve; CA: cardiac arrest; CASPRI: Cardiac arrest survival postresuscitation in-hospital; CPC: cerebral performance category; CPR: Cardiac pulmonary resuscitation; Cr: Creatinine; DBP: Diastolic blood pressure; Fib: Fibrinogen; GCS: Glasgow coma scale; Glu: Glucose; Hb: Hemoglobin; HCT: Hematocrit; HL: Hosmer-Lemeshow test; IHCA: In-hospital cardiac arrest; Lac: Lactate; MEWS: Modified early warning score; NPV: Negative predictive value; OHCA: Out-of-hospital cardiac arrest; PEA: Pulseless electrical activity; PPV: Positive predictive value; PT: Prothrombin time; PLT: Platelet; RBC: Red blood cell; ROC: Receiver operating characteristic curve; ROSC: Return of spontaneous circulation; SBP: Systolic blood pressure; SOFA: Sequential organ failure assessment; $\mathrm{SpO}_{2}$ : Saturation of pulse oxygen; RR: Respiration rate; TBIL: Total bilirubin; VF: Ventricular fibrillation; VT: Pulseless ventricular tachycardia; WBC: White blood cell.

\section{Acknowledgements}

None.

Funding

This study was supported financially by grants from 1.3.5 Project for Disciplines of Excellence, West China Hospital, Sichuan University (ZYJC18019).

\section{Author's contributions}

$\mathrm{BL}$ and $\mathrm{YH}$ contributed to the study concept and design. $\mathrm{PJ}, \mathrm{JS}, \mathrm{TH}, \mathrm{QL}$, and $\mathrm{RL}$ contributed to the date acquisition and analysis. All authors critically revised the manuscript. YC supervised the study.

Ethics approval and consent to participate

This study complied with the Declaration of Helsinki and was approved by China Ethics Committee of Registering Clinical Trials (chiECRT-20180032).

Consent for publication

Not applicable.

Competing interests

The authors declare that they have no competing interests.

\section{Author details}

${ }^{1}$ Department of Emergency Medicine, West China Hospital, Sichuan University, Guoxue Road 37, 610041 Chengdu, China. ${ }^{2}$ Diaster Medicine Center, Sichuan University, Guoxue Road 37, 610041 Chengdu, China. ${ }^{3}$ Laboratory of Emergency Medicine, West China Hospital, Sichuan University, Guoxue Road 37, 610041 Chengdu, China. ${ }^{4}$ West China School of Medicine, Sichuan University, Guoxue Road 37, 610041 Chengdu, China.

\section{References}

1. Thai, T.N., Ebell, M.H.: Prospective validation of the good outcome following attempted resuscitation (go-far) score for in-hospital cardiac arrest prognosis. Resuscitation 140, 2-8 (2019)

2. D'Arrigo, S., Cacciola, S., Dennis, M., Jung, C., Kagawa, E., Antonelli, M., Sandroni, C.: Predictors of favourable outcome after in-hospital cardiac arrest treated with extracorporeal cardiopulmonary resuscitation: a systematic review and meta-analysis. Resuscitation 121, 62-70 (2017)

3. Radeschi, G., Mina, A., Berta, G., Fassiola, A., Roasio, A., Urso, F., Penso, R., Zummo, U., Berchialla, P., Ristagno, G., et al.: Incidence and outcome of in-hospital cardiac arrest in italy: a multicentre observational study in the piedmont region. Resuscitation 119, 48-55 (2017)

4. Shao, F., Li, C.S., Liang, L.R., Qin, J., Ding, N., Fu, Y., Yang, K., Zhang, G.Q., Zhao, L., Zhao, B., et al.: Incidence and outcome of adult in-hospital cardiac arrest in beijing, china. Resuscitation 102, 51-56 (2016)

5. Herlitz, J., Engdahl, J., Svensson, L., Ängquist, K.-A., Young, M., Holmberg, S.: Factors associated with an increased chance of survival among patients suffering from an out-of-hospital cardiac arrest in a national perspective in sweden. American heart journal 149(1), 61-66 (2005)

6. Nolan, J.P., Soar, J., Cariou, A., Cronberg, T., Moulaert, V.R., Deakin, C.D., Bottiger, B.W., Friberg, H., Sunde, K., Sandroni, C.: European resuscitation council and european society of intensive care medicine 2015 guidelines for post-resuscitation care. Intensive care medicine 41(12), 2039-2056 (2015)

7. Sandroni, C., Cariou, A., Cavallaro, F., Cronberg, T., Friberg, H., Hoedemaekers, C., Horn, J., Nolan, J.P., Rossetti, A.O., Soar, J.: Prognostication in comatose survivors of cardiac arrest: an advisory statement from the european resuscitation council and the european society of intensive care medicine. Intensive care medicine 40(12), 1816-1831 (2014)

8. Wang, C.-H., Huang, C.-H., Chang, W.-T., Tsai, M.-S., Yu, P.-H., Wu, Y.-W., Chen, W.-J.: Prognostic performance of simplified out-of-hospital cardiac arrest (ohca) and cardiac arrest hospital prognosis (cahp) scores in an east asian population: A prospective cohort study. Resuscitation 137, 133-139 (2019)

9. Knaus, W.A., Draper, E.A., Wagner, D.P., Zimmerman, J.E.: Apache ii: a severity of disease classification system. Critical care medicine 13(10), 818-829 (1985)

10. Vincent, J.-L., Moreno, R., Takala, J., Willatts, S., De Mendonça, A., Bruining, H., Reinhart, C., Suter, P., Thijs, L.G.: The SOFA (Sepsis-related Organ Failure Assessment) score to describe organ dysfunction/failure. Springer (1996) 
11. Subbe, C., Kruger, M., Rutherford, P., Gemmel, L.: Validation of a modified early warning score in medical admissions. Qjm 94(10), 521-526 (2001)

12. Akinpelu, O.V., Waissbluth, S., Daniel, S.J.: Auditory risk of hyperbilirubinemia in term newborns: a systematic review. International journal of pediatric otorhinolaryngology 77(6), 898-905 (2013)

13. Falcao, A.S., Silva, R.F., Vaz, A.R., Gomes, C., Fernandes, A., Barateiro, A., Tiribelli, C., Brites, D.: Cross-talk between neurons and astrocytes in response to bilirubin: adverse secondary impacts. Neurotoxicity research 26(1), 1-15 (2014)

14. Waterham, M., Bhatia, R., Donath, S., Molesworth, C., Tan, K., Stewart, M.: Phototherapy in transport for neonates with unconjugated hyperbilirubinaemia. Journal of paediatrics and child health 52(1), 67-71 (2016)

15. Ye, H.-B., Shi, H.-B., Wang, J., Ding, D.-L., Yu, D.-Z., Chen, Z.-N., Li, C.-Y., Zhang, W.-T., Yin, S.-K.: Bilirubin induces auditory neuropathy in neonatal guinea pigs via auditory nerve fiber damage. Journal of neuroscience research 90(11), 2201-2213 (2012)

16. Loftspring, M.C., Johnson, H.L., Feng, R., Johnson, A.J., Clark, J.F.: Unconjugated bilirubin contributes to early inflammation and edema after intracerebral hemorrhage. Journal of Cerebral Blood Flow \& Metabolism 31(4), 1133-1142 (2011)

17. Gul, M., Uyarel, H., Ergelen, M., Akgul, O., Karaca, G., Turen, S., Ugur, M., Ertürk, M., Kul, S., Surgit, O., et al.: Prognostic value of total bilirubin in patients with st-segment elevation acute myocardial infarction undergoing primary coronary intervention. The American journal of cardiology 111(2), 166-171 (2013)

18. Leite, H.P., da Silva, A.V.R., de Oliveira Iglesias, S.B., Nogueira, P.C.K.: Serum albumin is an independent predictor of clinical outcomes in critically ill children. Pediatric Critical Care Medicine 17(2), 50-57 (2016)

19. Vincent, J.-L., Dubois, M.-J., Navickis, R.J., Wilkes, M.M.: Hypoalbuminemia in acute illness: is there a rationale for intervention?: a meta-analysis of cohort studies and controlled trials. Annals of surgery 237(3), 319 (2003)

20. Matsuyama, T., Iwami, T., Yamada, T., Hayakawa, K., Yoshiya, K., Irisawa, T., Abe, Y., Nishimura, T., Uejima, T., Ohishi, Y., et al.: Effect of serum albumin concentration on neurological outcome after out-of-hospital cardiac arrest (from the critical [comprehensive registry of intensive cares for ohca survival] study in osaka, japan). The American journal of cardiology 121(2), 156-161 (2018)

21. Larkin, C.M., Hante, N.K., Breen, E.P., Tomaszewski, K.A., Eisele, S., Radomski, M.W., Ryan, T.A., Santos-Martinez, M.-J.: Role of matrix metalloproteinases 2 and 9, toll-like receptor 4 and platelet-leukocyte aggregate formation in sepsis-associated thrombocytopenia. PloS one 13(5) (2018)

22. Hayman, E.G., Patel, A.P., Kimberly, W.T., Sheth, K.N., Simard, J.M.: Cerebral edema after cardiopulmonary resuscitation: a therapeutic target following cardiac arrest? Neurocritical care 28(3), 276-287 (2018)

23. Saghafinia, M., Motamedi, M.H.K., Piryaie, M., Rafati, H., Saghafi, A., Jalali, A., Madani, S.J., Kolahdehi, R.B.: Survival after in-hospital cardiopulmonary resuscitation in a major referral center. Saudi journal of anaesthesia 4(2), 68 (2010)

24. Gomes, A., Timerman, A., Souza, C., Mendes, C., Póvoas, H.F., Oliveira, A.d., Souza, J.: Prognostic factors of survival in post-cardiopulmonary-cerebral resuscitation in general hospital. Arquivos brasileiros de cardiologia 85(4), 262-271 (2005)

25. Wang, J., Ma, Q., Zhang, H., Liu, S., Zheng, Y.: Predictors of survival and neurologic outcome for adults with extracorporeal cardiopulmonary resuscitation: A systemic review and meta-analysis. Medicine 97(48) (2018)

26. Mehta, C., Brady, W.: Pulseless electrical activity in cardiac arrest: electrocardiographic presentations and management considerations based on the electrocardiogram. The American journal of emergency medicine 30(1), 236-239 (2012)

27. Cummins, R.O., Chamberlain, D., Hazinski, M.F., Nadkarni, V., Kloeck, W., Kramer, E., Becker, L., Robertson, C., Koster, R., Zaritsky, A., et al.: Recommended guidelines for reviewing, reporting, and conducting research on in-hospital resuscitation: The in-hospital 'utstein style' a statement for healthcare professionals from the american heart association, the european resuscitation council, the heart and stroke foundation of canada, the australian resuscitation council, and the resuscitation councils of southern africa. Circulation 95(8), 2213-2239 (1997)

28. Nolan, J.P., Soar, J., Smith, G.B., Gwinnutt, C., Parrott, F., Power, S., Harrison, D.A., Nixon, E., Rowan, K., et al:: Incidence and outcome of in-hospital cardiac arrest in the united kingdom national cardiac arrest audit. Resuscitation 85(8), 987-992 (2014)

29. Nadkarni, V.M., Larkin, G.L., Peberdy, M.A., Carey, S.M., Kaye, W., Mancini, M.E., Nichol, G., Lane-Truitt, T., Potts, J., Ornato, J.P., et al.: First documented rhythm and clinical outcome from in-hospital cardiac arrest among children and adults. Jama 295(1), 50-57 (2006)

30. Meaney, P.A., Nadkarni, V.M., Kern, K.B., Indik, J.H., Halperin, H.R., Berg, R.A.: Rhythms and outcomes of adult in-hospital cardiac arrest. Critical care medicine 38(1), 101-108 (2010)

31. Cooper, S., Cade, J.: Predicting survival, in-hospital cardiac arrests: resuscitation survival variables and training effectiveness. Resuscitation 35(1), 17-22 (1997)

32. Kaki, A.M., Alghalayini, K.W., Alama, M.N., Almazroaa, A.A., Khathlan, N.A.A., Sembawa, H., Ouseph, B.M.: An audit of in-hospital cardiopulmonary resuscitation in a teaching hospital in saudi arabia: A retrospective study. Saudi journal of anaesthesia 11(4), 415 (2017)

33. Sandroni, C., Nolan, J., Cavallaro, F., Antonelli, M.: In-hospital cardiac arrest: incidence, prognosis and possible measures to improve survival. Intensive care medicine 33(2), 237-245 (2007)

34. Dell'Anna, A.M., Sandroni, C., Lamanna, I., Belloni, I., Donadello, K., Creteur, J., Vincent, J.-L., Taccone, F.S.: Prognostic implications of blood lactate concentrations after cardiac arrest: a retrospective study. Annals of intensive care $7(1), 101$ (2017)

35. Zhou, B.-C., Zhang, Z., Zhu, J.-J., Liu, L.-J., Liu, C.-F.: Blood lactate or lactate clearance: Which is robust to predict the neurological outcomes after cardiac arrest? a systematic review and meta-analysis. BioMed research international 2018 (2018)

36. Khurana, D., Deoke, S.A.: Thrombocytopenia in critically ill patients: Clinical and laboratorial behavior and its correlation with short-term outcome during hospitalization. Indian journal of critical care medicine: 
peer-reviewed, official publication of Indian Society of Critical Care Medicine 21(12), 861 (2017)

37. Akca, S., Haji-Michael, P., De Mendonça, A., Suter, P., Levi, M., Vincent, J.-L.: Time course of platelet counts in critically ill patients. Critical care medicine 30(4), 753-756 (2002)

38. Levi, M.: Platelets in critical illness. In: Seminars in Thrombosis and Hemostasis, vol. 42, pp. $252-257$ (2016). Thieme Medical Publishers

39. Buchtele, N., Schober, A., Schoergenhofer, C., Spiel, A., Mauracher, L., Weiser, C., Sterz, F., Jilma, B., Schwameis, M.: Added value of the dic score and of d-dimer to predict outcome after successfully resuscitated out-of-hospital cardiac arrest. European journal of internal medicine 57, 44-48 (2018)

40. Patel, M.J., Khan, N.U., Furqan, M., Awan, S., Khan, M.S., Kashif, W., Sorathia, A.L., Hussain, S.A., Mir, M.U.: Apache ii scores as predictors of cardio pulmonary resuscitation outcome: Evidence from a tertiary care institute in a low-income country. Saudi journal of anaesthesia 6(1), 31 (2012)

41. Donnino, M.W., Salciccioli, J.D., Dejam, A., Giberson, T., Giberson, B., Cristia, C., Gautam, S., Cocchi, M.N.: Apache ii scoring to predict outcome in post-cardiac arrest. Resuscitation 84(5), 651-656 (2013)

42. Ferreira, F.L., Bota, D.P., Bross, A., Mélot, C., Vincent, J.-L.: Serial evaluation of the sofa score to predict outcome in critically ill patients. Jama 286(14), 1754-1758 (2001)

43. Cour, M., Bresson, D., Hernu, R., Argaud, L.: Sofa score to assess the severity of the post-cardiac arrest syndrome. Resuscitation 102, 110-115 (2016)

44. Zein, J.G., Love, T.E., Erzurum, S.C.: Asthma is associated with a lower risk of sepsis and sepsis-related mortality. American journal of respiratory and critical care medicine 196(6), 787-790 (2017)

45. DeVoe, B., Roth, A., Maurer, G., Tamuz, M., Lesser, M., Pekmezaris, R., Makaryus, A.N., Hartman, A., DiMarzio, P.: Correlation of the predictive ability of early warning metrics and mortality for cardiac arrest patients receiving in-hospital advanced cardiovascular life support. Heart \& Lung 45(6), 497-502 (2016)

46. De Meester, K., Das, T., Hellemans, K., Verbrugghe, W., Jorens, P., Verpooten, G., Van Bogaert, P.: Impact of a standardized nurse observation protocol including mews after intensive care unit discharge. Resuscitation 84(2), 184-188 (2013)

Figures

Figure 1 Flow of patients.

Figure 2 The efficacy of independent risk factors for poor neurological prognosis in ROSC patient. a Resuscitation time, Non-shockable rhythm, Non-cardiogenic etiology, total bilirubin, lactic acid. b Albumin. Abbreviations: TBIL total bilirubin, Lac lactic acid during resuscitation. According to ROC curve analysis: TBIL $(A U C=0.666)$, albumin $(A U C=0.737)$, Lac $(A \cup C=0.760)$, resuscitation time $(A \cup C=0.748)$ non-shockable rhythms $(0.621)$, and non-cardiogenic etiologies $(A U C=0.640)$. The cutoff values are TBIL $(23.4 \mu \mathrm{mol} / \mathrm{L}), \operatorname{ALB}(34.5$ $\mathrm{g} / \mathrm{L})$, Lac $(5.3 \mathrm{mmol} / \mathrm{L})$, and resuscitation time $(9 \mathrm{~min})$.

Figure 3 The efficacy of CASPRI score and other scores in evaluating the prognosis of ROSC patients. CASPRI score $(A \cup C=0.896)$, sOHCA score $(A \cup C=0.829)$, SOFA score $(A \cup C=0.815)$, APACHE II score $(A \cup C=0.752)$, , and MEWS score $(A \cup C=0.599)$.

Figure 4 Comparison of poor neurological prognosis rate in CASPRI predicted high-, intermediate-, and low-risk groups. High-risk group (HRG), intermediate-risk group (MRG), and low-risk group (LRG).

Figure 5 Verifying the efficacy of the CASPRI score in evaluating the neurological prognosis of ROSC patients. CASPRI score $(A \cup C=0.831)$, SOFA score $(A \cup C=0.726)$, APACHE II score $(A \cup C=0.789)$, MEWS score $(A \cup C=0.693)$, and sOHCA score $(A \cup C=0.791)$. 
Table 1 Comparison of baseline information between test group and validation group. Abbreviations: T temperature, HR heart rate, SBP systolic blood pressure, DBP diastolic blood pressure, RR respiratory rate, $\mathrm{SpO}_{2}$ oxygen saturation, $\mathrm{RBC}$ red blood cell, WBC white blood cell, TBIL total bilirubin, ALT alanine aminotransferase, AST aspartate transaminase, ALB albumin, PT prothrombin time, APTT activated partial thromboplastin time, Lac lactic acid during resuscitation.

\begin{tabular}{|c|c|c|c|}
\hline & Test group & Validation group & P value \\
\hline Gender & & & 0.873 \\
\hline Male & $323(64.21 \%)$ & $166(64.84 \%)$ & \\
\hline Female & $180(35.79 \%)$ & $90(35.16 \%)$ & \\
\hline Age & $60.11 \pm 18.62$ & $60.26 \pm 17.19$ & 0.635 \\
\hline Shockable rhythm & & & 0.895 \\
\hline Yes & $46(9.15 \%)$ & $24(9.38 \%)$ & \\
\hline No & $457(90.85 \%)$ & $232(90.62 \%)$ & \\
\hline Cardiogenic etiology & & & 0.62 \\
\hline Yes & $89(17.69 \%)$ & $49(19.14 \%)$ & \\
\hline No & $414(82.31 \%)$ & $207(80.86 \%)$ & \\
\hline \multicolumn{4}{|l|}{ Type of disease } \\
\hline Respiratory system & $131(26.04 \%)$ & $63(24.61 \%)$ & \\
\hline Circulatory system & $104(20.68 \%)$ & $49(19.14 \%)$ & \\
\hline Gastrointestinal system & $137(27.24 \%)$ & $58(22.66 \%)$ & \\
\hline \multicolumn{4}{|l|}{ During emergency room visit } \\
\hline $\mathrm{T}\left({ }^{\circ} \mathrm{C}\right)$ & $36.82 \pm 1.00$ & $36.68 \pm 0.65$ & 0.06 \\
\hline $\mathrm{HR}(\mathrm{bpm})$ & $105.70 \pm 30.06$ & $104.59 \pm 30.27$ & 0.732 \\
\hline $\mathrm{SBP}(\mathrm{mmHg})$ & $117.50 \pm 29.19$ & $116.13 \pm 31.81$ & 0.441 \\
\hline $\mathrm{DBP}(\mathrm{mmHg})$ & $70.5 \pm 20.6$ & $68.53 \pm 21.90$ & 0.248 \\
\hline $\mathrm{RR}(\mathrm{bpm})$ & $23.19 \pm 5.66$ & $23.70 \pm 7.70$ & 0.644 \\
\hline $\mathrm{SpO}_{2}(\%)$ & $91.88 \pm 9.10$ & $92.31 \pm 8.17$ & 0.488 \\
\hline \multicolumn{4}{|l|}{ First test at emergency department } \\
\hline $\operatorname{RBC}\left(10^{12} / \mathrm{L}\right)$ & $3.92(2.88,4.58)$ & $3.76(2.71,4.49)$ & 0.142 \\
\hline Hemoglobin $(\mathrm{g} / \mathrm{L})$ & $116.5(84.0,135.0)$ & $110.0(82.0,131.0)$ & 0.147 \\
\hline Platelet $\left(10^{9} / \mathrm{L}\right)$ & $143.5(85.0,214.0)$ & $135.5(82.0,194.0)$ & 0.406 \\
\hline WBC $\left(10^{9} / \mathrm{L}\right)$ & $12.44(8.13,18.69)$ & $11.8(7.52,17.74)$ & 0.253 \\
\hline TBIL $(\mu \mathrm{mol} / \mathrm{L})$ & $14.85(8.30,28.40)$ & $14.80(9.10,29.50)$ & 0.754 \\
\hline $\mathrm{ALT}(\mathrm{IU} / \mathrm{L})$ & $34.0(17.0,83.0)$ & $31.0(16.0,81.0)$ & 0.326 \\
\hline AST (IU/L) & $52.0(28.0,168.0)$ & $50.5(23.0,138.0)$ & 0.123 \\
\hline $\operatorname{ALB}(\mathrm{g} / \mathrm{L})$ & $32.24 \pm 7.97$ & $32.14 \pm 8.44$ & 0.783 \\
\hline Blood glucose $(\mathrm{mmol} / \mathrm{L})$ & $8.57(6.36,12.75)$ & $8.41(6.25,13.14)$ & 0.838 \\
\hline Creatinine $(\mu \mathrm{mol} / \mathrm{L})$ & $117.0(78.0,216.0)$ & $118.0(73.0,211.0)$ & 0.589 \\
\hline $\mathrm{Na}^{+}(\mathrm{mmol} / \mathrm{L})$ & $136.65 \pm 7.55$ & $137.73 \pm 6.88$ & 0.074 \\
\hline $\mathrm{K}^{+}(\mathrm{mmol} / \mathrm{L})$ & $4.20 \pm 1.04$ & $4.34 \pm 1.46$ & 0.097 \\
\hline PT (s) & $14.90(13.10,18.90)$ & $15.65(13.00,20.50)$ & 0.339 \\
\hline APTT (s) & $38.20(30.10,52.60)$ & $37.50(30.30,55.20)$ & 0.876 \\
\hline D-dimer $(\mathrm{mg} / \mathrm{L})$ & $4.72(1.77,12.00)$ & $4.70(1.75,12.34)$ & 0.928 \\
\hline Resuscitation time (min) & $10(4,20)$ & $11(4,18)$ & 0.925 \\
\hline $\mathrm{Lac}(\mathrm{mmol} / \mathrm{L})$ & $6.75(3.30,13.50)$ & $6.10(2.40,11.10)$ & 0.066 \\
\hline Number of cases with good prognosis & $59(11.73 \%)$ & $38(14.84 \%)$ & 0.25 \\
\hline
\end{tabular}


Table 2 Comparison of general information, laboratory tests, APACHE II, SOFA, MEWS and sOHCA scores between favorable and unfavorable prognosis group. ${ }^{*}$ for $\mathrm{p}<0.05$. Abbreviations: See Table 1.

\begin{tabular}{|c|c|c|c|}
\hline & Favorable prognosis group $(n=444)$ & Unfavorable prognosis group $(n=59)$ & $P$ value \\
\hline Gender & & & 0.57 \\
\hline Male & $284(61.2 \%)$ & $39(66.1 \%)$ & \\
\hline Female & $180(38.8 \%)$ & $20(33.9 \%)$ & \\
\hline Age & $59.98 \pm 21.67$ & $60.12 \pm 18.21$ & 0.648 \\
\hline Shockable rhythm & & & $0.000 *$ \\
\hline Yes & $28(6.3 \%)$ & $18(30.5 \%)$ & \\
\hline No & $416(93.7 \%)$ & $41(69.5 \%)$ & \\
\hline Cardiogenic etiology & & & $0.000 *$ \\
\hline Yes & $62(14.0 \%)$ & $25(42.4 \%)$ & \\
\hline No & $382(86.0 \%)$ & $34(57.6 \%)$ & \\
\hline \multicolumn{4}{|l|}{ During emergency room visit } \\
\hline $\mathrm{T}\left({ }^{\circ} \mathrm{C}\right)$ & $36.83 \pm 1.04$ & $36.76 \pm 0.76$ & 0.208 \\
\hline $\mathrm{HR}(\mathrm{bpm})$ & $106.66 \pm 28.64$ & $98.41 \pm 38.77$ & $0.022 *$ \\
\hline $\mathrm{SBP}(\mathrm{mmHg})$ & $115.99 \pm 28.63$ & $128.97 \pm 31.06$ & $0.003^{*}$ \\
\hline $\mathrm{DBP}(\mathrm{mmHg})$ & $69.70 \pm 20.35$ & $76.38 \pm 21.98$ & 0.05 \\
\hline $\mathrm{RR}(\mathrm{bpm})$ & $23.43 \pm 5.76$ & $21.43 \pm 4.50$ & $0.002 *$ \\
\hline $\mathrm{SpO}_{2}(\%)$ & $91.53 \pm 9.30$ & $94.56 \pm 6.99$ & $0.000^{*}$ \\
\hline Resuscitation time (min) & $11(5,21)$ & $5(2,8)$ & $0.000 *$ \\
\hline \multicolumn{4}{|c|}{ First test at emergency department } \\
\hline $\operatorname{RBC}\left(10^{12} / \mathrm{L}\right)$ & $3.88(2.84,4.55)$ & $4.16(3.46,4.76)$ & $0.019 *$ \\
\hline Hemoglobin $(\mathrm{g} / \mathrm{L})$ & $114.0(82.0,134.0)$ & $124.0(100.0,141.0)$ & $0.020 *$ \\
\hline Hematocrit $(\mathrm{L} / \mathrm{L})$ & $0.35(0.25,0.41)$ & $0.38(0.29,0.43)$ & $0.022^{*}$ \\
\hline Platelet $\left(10^{9} / \mathrm{L}\right)$ & $140.5(78.5,206.5)$ & $193.5(118.0,258.0)$ & $0.000 *$ \\
\hline WBC $\left(10^{9} / \mathrm{L}\right)$ & $12.66(8.13,19.17)$ & $10.84(8.33,16.43)$ & 0.29 \\
\hline TBIL $(\mu \mathrm{mol} / \mathrm{L})$ & $15.70(8.75,31.78)$ & $9.4(6.4,16.8)$ & $0.000^{*}$ \\
\hline $\mathrm{ALT}(\mathrm{IU} / \mathrm{L})$ & $34.0(17.0,91.0)$ & $36.5(15.0,67.0)$ & $0.301 *$ \\
\hline AST (IU'L) & $52.0(28.5,194.5)$ & $44.0(27.0,94.0)$ & 0.099 \\
\hline $\operatorname{ALB}(\mathrm{g} / \mathrm{L})$ & $31.53 \pm 7.94$ & $37.65(5.92)$ & $0.000 *$ \\
\hline Blood glucose $(\mathrm{mmol} / \mathrm{L})$ & $8.56(6.28,13.01)$ & $8.65(6.86,12.01)$ & 0.778 \\
\hline Creatinine $(\mu \mathrm{mol} / \mathrm{L})$ & $118.5(78.0,224.0)$ & $101.0(68.0,166.0)$ & 0.095 \\
\hline $\mathrm{Na}^{+}(\mathrm{mmol} / \mathrm{L})$ & $136.65 \pm 7.61$ & $136.67 \pm 7.21$ & 0.599 \\
\hline $\mathrm{K}^{+}(\mathrm{mmol} / \mathrm{L})$ & $4.19 \pm 1.03$ & $4.31 \pm 1.40$ & 0.866 \\
\hline $\mathrm{PT}(\mathrm{s})$ & $15.20(13.25,19.25)$ & $13.15(11.90,14.40)$ & $0.000 *$ \\
\hline $\operatorname{APTT}(s)$ & $39.60(31.55,55.10)$ & $29.25(26.00,36.20)$ & $0.000 *$ \\
\hline D-dimer (mg/L) & $5.42(1.93,14.19)$ & $2.09(0.89,4.54)$ & $0.000^{*}$ \\
\hline $\mathrm{Lac}(\mathrm{mmol} / \mathrm{L})$ & $7.55(3.80,14.10)$ & $2.90(1.80,4.80)$ & $0.000 *$ \\
\hline sOHCA score & $26.85(16.22,36.91)$ & $5.85(-6.79,17.08)$ & $0.000 *$ \\
\hline APACHE II score & $26.0(23.0,30.0)$ & $19.5(15.0,26.0)$ & $0.000 *$ \\
\hline SOFA score & $11.0(9.0,13.0)$ & $7.0(5.0,9.0)$ & $0.000 *$ \\
\hline MEWS score & $4.0(2.0,5.0)$ & $3.0(2.0,5.0)$ & $0.000 *$ \\
\hline
\end{tabular}

Table 3 Screening of independent risk factors for poor neurological outcomes in ROSC patients. * for $\mathrm{p}<0.05$. Abbreviations: $\mathrm{HR}$ heart rate, $\mathrm{SBP}$ systolic blood pressure, $\mathrm{RR}$ respiratory rate, $\mathrm{SpO}_{2}$ oxygen saturation, PLT platelet count, TBIL total bilirubin, AST aspartate transaminase, ALB albumin, PT prothrombin time, APTT activated partial thromboplastin time, Lac lactic acid during resuscitation.

\begin{tabular}{lllllllll}
\hline \multirow{2}{*}{ Variants } & \multicolumn{3}{c}{ Univariate analysis } & \multicolumn{4}{c}{ Multivariate analysis } \\
\cline { 2 - 9 } & \multicolumn{1}{c}{ OR } & \multicolumn{1}{c}{$95 \% \mathrm{Cl}$} & \multicolumn{1}{c}{$\mathrm{P}$} & \multicolumn{1}{c}{$\beta$} & \multicolumn{1}{c}{ OR } & \multicolumn{1}{c}{$95 \% \mathrm{Cl}$} & $\mathrm{P}$ \\
\hline Resuscitation time & 0.096 & 0.909 & $0.896-0.950$ & $0.000^{*}$ & 0.088 & 1.092 & $1.041-1.146$ & $0.000^{*}$ \\
HR & 0.01 & 1.01 & $1.000-1.019$ & $0.040^{*}$ & -0.008 & 0.992 & $0.979-1.005$ & 0.24 \\
SBP & -0.015 & 0.986 & $0.977-0.994$ & $0.001^{*}$ & -0.006 & 0.994 & $0.981-1.007$ & 0.345 \\
RR & 0.069 & 1.017 & $1.013-1.133$ & $0.016^{*}$ & 0.05 & 1.051 & $0.972-1.137$ & 0.212 \\
SpO2 & -0.056 & 0.946 & $0.904-0.989$ & $0.015^{*}$ & -0.022 & 0.978 & $0.932-1.026$ & 0.36 \\
Non-shockable rhythm & 1.875 & 6.523 & $3.326-12.791$ & $0.000^{*}$ & 2.138 & 8.482 & $2.837-25.358$ & $0.000^{*}$ \\
Non-cardiogenic etiology & 1.474 & 4.366 & $2.444-7.800$ & $0.000^{*}$ & 1.043 & 2.838 & $1.212-6.640$ & $0.016^{*}$ \\
PLT & -0.004 & 0.996 & $0.994-0.998$ & $0.000^{*}$ & -0.003 & 0.997 & $0.994-1.000$ & 0.074 \\
TBIL & 0.048 & 1.049 & $1.018-1.081$ & $0.002^{*}$ & 0.055 & 1.056 & $1.010-1.104$ & $0.016^{*}$ \\
AST & 0.002 & 1.002 & $1.000-1.004$ & $0.043^{*}$ & 0 & 1 & $0.998-1.002$ & 0.895 \\
ALB & -0.103 & 0.902 & $0.868-0.937$ & $0.000^{*}$ & -0.104 & 0.901 & $0.852-0.953$ & $0.000^{*}$ \\
PT & 0.063 & 1.065 & $1.008-1.125$ & $0.025^{*}$ & -0.035 & 0.966 & $0.931-1.002$ & 0.062 \\
APTT & 0.045 & 1.046 & $1.021-1.071$ & $0.000^{*}$ & 0.014 & 1.014 & $0.990-1.038$ & 0.258 \\
D-dimer & 0.097 & 1.102 & $1.044-1.163$ & $0.000^{*}$ & 0.044 & 1.045 & $0.991-1.101$ & 0.103 \\
Lac & 0.211 & 1.235 & $1.140-1.338$ & $0.000^{*}$ & 0.2 & 1.222 & $1.112-1.343$ & $0.000^{*}$ \\
\hline
\end{tabular}


Table 4 Efficacy analysis of independent risk factors for the poor prognosis in ROSC patients. Abbreviations: TBIL total bilirubin, ALB albumin, Lac lactic acid during resuscitation.

\begin{tabular}{lllllllll}
\hline & AUC & Cut-off value & Sensitivity $(\%)$ & Specificity $(\%)$ & PPV $(\%)$ & NPV $(\%)$ & + LR & -LR \\
\hline TBIL & 0.666 & $24.6(\mu \mathrm{mol} / \mathrm{L})$ & 32.8 & 94.92 & 97.9 & 15.7 & 6.35 & 0.72 \\
ALB & 0.737 & $34.5(\mathrm{~g} / \mathrm{L})$ & 64.86 & 76.27 & 95.4 & 22.4 & 22.4 & 0.46 \\
Lac & 0.76 & $5.3(\mathrm{mmol} / \mathrm{L})$ & 63.06 & 79.66 & 95.9 & 22.3 & 22.3 & 0.46 \\
Resuscitation time & 0.747 & $9(\mathrm{~min})$ & 54.73 & 84.75 & 96.4 & 19.9 & 19.9 & 0.53 \\
\hline
\end{tabular}

Table 5 Multivariate regression results of selected variables in the test group. ${ }^{*}$ for $p<0.05$. Abbreviations: ALB albumin, TBIL total bilirubin, Lac lactic acid during resuscitation.

\begin{tabular}{|c|c|c|c|c|c|}
\hline & $\beta$ & Scored & OR & $95 \% \mathrm{Cl}$ & $\mathrm{P}$ \\
\hline \multicolumn{6}{|l|}{$\operatorname{ALB}(\mathrm{g} / \mathrm{L})$} \\
\hline$>34.5$ & 1 & 0 & & $(2419,10821)$ & $0.000 *$ \\
\hline$\leq 34.5$ & 1.633 & 2 & 5.117 & $(2.419,10.821)$ & $0.0000^{2}$ \\
\hline \multicolumn{6}{|c|}{ TBIL $(\mu \mathrm{mol} / \mathrm{L})$} \\
\hline$\leq 23.6$ & 1 & 0 & 11531 & $(2833 \quad 46940)$ & $0 \cap 01 *$ \\
\hline$>23.6$ & 2.445 & 3 & 11.531 & $(2.833,40.940)$ & 0.001 \\
\hline \multicolumn{6}{|c|}{$\mathrm{Lac}(\mathrm{mmol} / \mathrm{L})$} \\
\hline$\leq 5.3$ & 1 & 0 & 5.449 & $(2.539,11.694)$ & $0.000 *$ \\
\hline \multicolumn{4}{|c|}{ Resuscitation time ( $\mathrm{min})$} & & \\
\hline$>9$ & 1 & 0 & & & \\
\hline$\leq 9$ & 2.189 & 2 & 8.929 & $(3.807,20.943)$ & $0.000^{*}$ \\
\hline \multicolumn{6}{|c|}{ Shockable rhythm } \\
\hline Yes & 1 & 0 & 6859 & $(2559 \quad 18387)$ & $0000 *$ \\
\hline No & 1.926 & 2 & 0.039 & $(2.509,10.501)$ & \\
\hline \multicolumn{6}{|c|}{ Cardiogenic etiology } \\
\hline Yes & 1 & $\begin{array}{l}0 \\
1\end{array}$ & 2.517 & $(1.135,5.584)$ & $0.023 *$ \\
\hline
\end{tabular}

Table 6 Assessment of poor prognosis of ROSC patients with different scoring system. ${ }^{*}$ for $\mathrm{p}<0.05$.

\begin{tabular}{llllll}
\hline & AUC & SE & Z & P & $95 \% \mathrm{Cl}$ \\
\hline CASPRI & 0.896 & 0.023 & 17.453 & $0.000^{*}$ & $0.851,0.941$ \\
APACHE II & 0.752 & 0.036 & 6.983 & $0.000^{*}$ & $0.682,0.822$ \\
SOFA & 0.815 & 0.028 & 11.348 & $0.000^{*}$ & $0.778,0.848$ \\
MEWS & 0.599 & 0.041 & 2.421 & $0.016^{*}$ & $0.518,0.680$ \\
SOHCA & 0.829 & 0.023 & 14.1 & $0.000^{*}$ & $0.784,0.875$ \\
\hline
\end{tabular}

Table 7 Comparison of the efficacy of different scoring system in evaluating the prognosis of ROSC patients. * for $\mathrm{p}<0.05$.

\begin{tabular}{llllll}
\hline & $\Delta \mathrm{A}$ & $\mathrm{SE}$ & $\mathrm{Z}$ & $\mathrm{P}$ & $95 \% \mathrm{Cl}$ \\
\hline CASPRI \& APACHE II & 0.144 & 0.035 & 3.139 & $<0.001^{*}$ & $0.076,0.212$ \\
CASPRI \& SOFA & 0.084 & 0.034 & 2.478 & $0.013^{*}$ & $0.018,0.150$ \\
CASPRI \& MEWS & 0.297 & 0.041 & 7.188 & $<0.001^{*}$ & $0.216,0.378$ \\
CASPRI \& SOHCA & 0.067 & 0.028 & 2.405 & $0.016^{*}$ & $0.012,0.121$ \\
\hline
\end{tabular}

Table 8 Verification of the efficacy of the CASPRI score in evaluating the prognosis of ROSC patients.

\begin{tabular}{llllll}
\hline & AUC & SE & Z & P & $95 \% \mathrm{Cl}$ \\
\hline CASPRI & 0.831 & 0.031 & 10.841 & $0.000^{*}$ & $0.780,0.875$ \\
APACHE II & 0.789 & 0.042 & 6.833 & $0.000^{*}$ & $0.734,0.837$ \\
SOFA & 0.726 & 0.042 & 5.377 & $0.000^{*}$ & $0.667,0.780$ \\
MEWS & 0.693 & 0.044 & 4.42 & $0.000^{*}$ & $0.632,0.749$ \\
SOHCA & 0.791 & 0.041 & 7.153 & $0.000^{*}$ & $0.736,0.839$ \\
\hline
\end{tabular}

Table 9 Comparison of the efficacy of CASPRI and other scoring systems in evaluating the prognosis of ROSC patients in the validation group.

\begin{tabular}{lll}
\hline & $\Delta \mathrm{A}$ & $\mathrm{SE}$ \\
\hline CASPRI \& APACHE II & 0.042 & 0.044 \\
CASPRI \& SOFA & 0.105 & 0.049 \\
CASPRI \& MEWS & 0.139 & 0.05 \\
CASPRI \& SOHCA & 0.046 & 0.042 \\
\hline
\end{tabular}


Figures

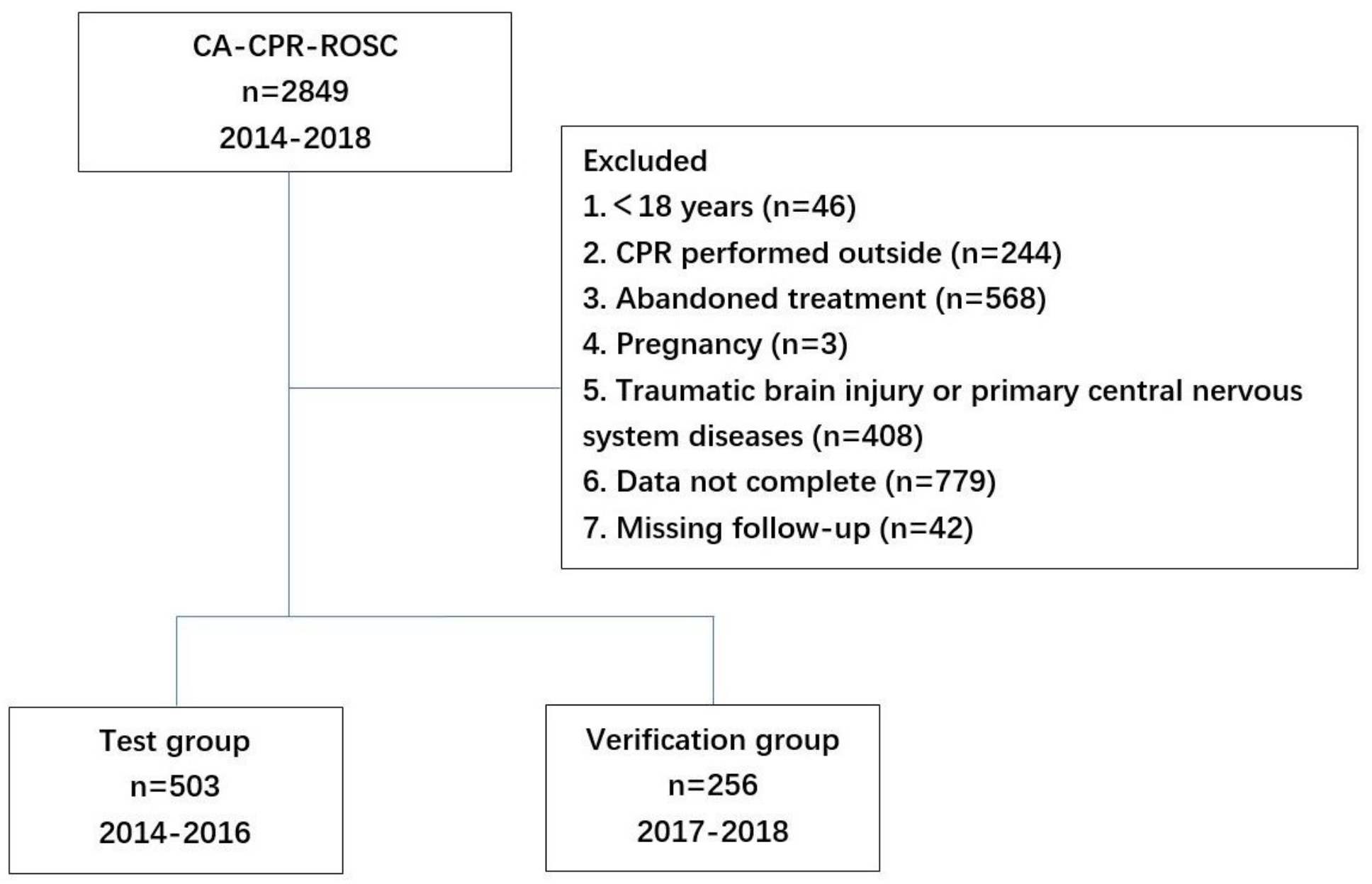

Figure 1

Flow of patients.
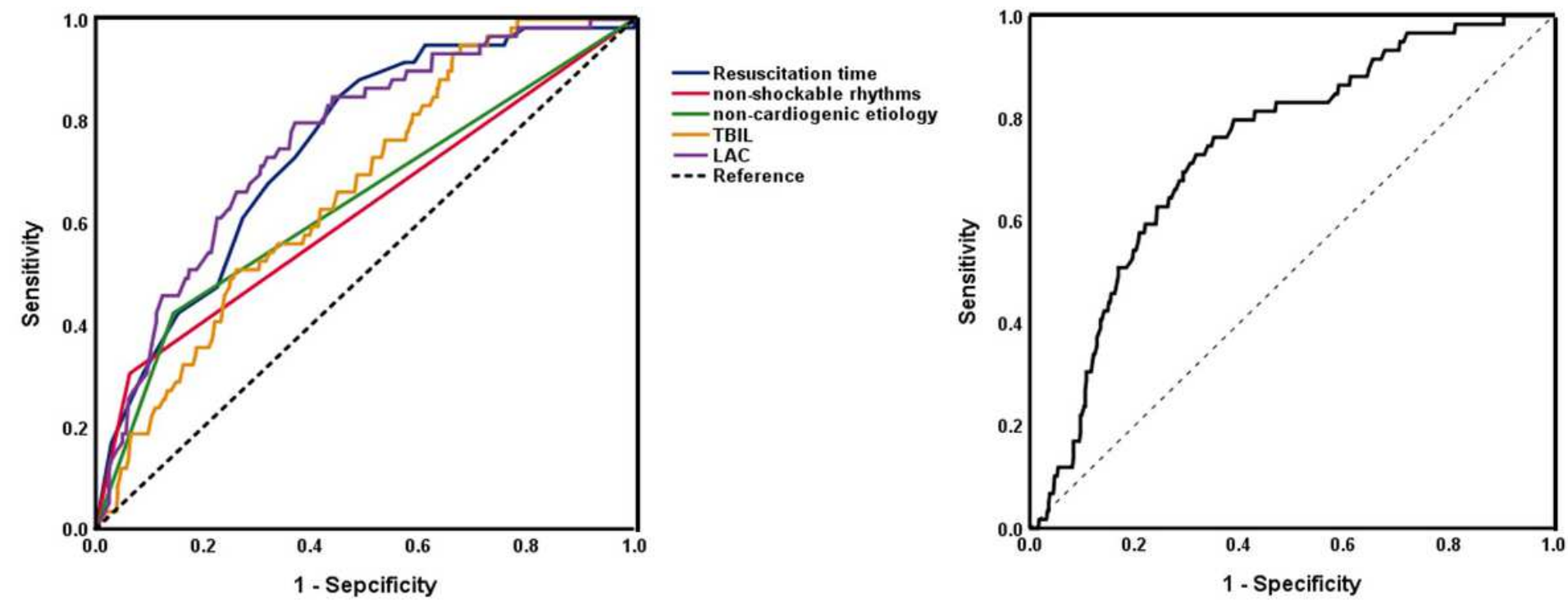

Figure 2 
The efficacy of independent risk factors for poor neurological prognosis in ROSC patient. a Resuscitation time, Non-shockable rhythm, Non-cardiogenic etiology, total bilirubin, lactic acid. b Albumin.

Abbreviations: TBIL total bilirubin, Lac lactic acid during resuscitation. According to ROC curve analysis:

TBIL (AUC=0.666), albumin (AUC=0.737), Lac (AUC=0.760), resuscitation time (AUC=0.748)non-

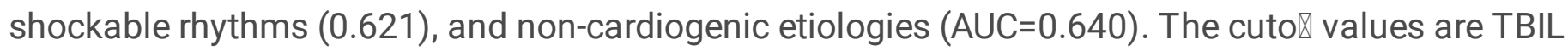
(23.4凶mol/L), ALB (34.5 g/L), Lac (5.3 mmol/L), and resuscitation time (9 min).

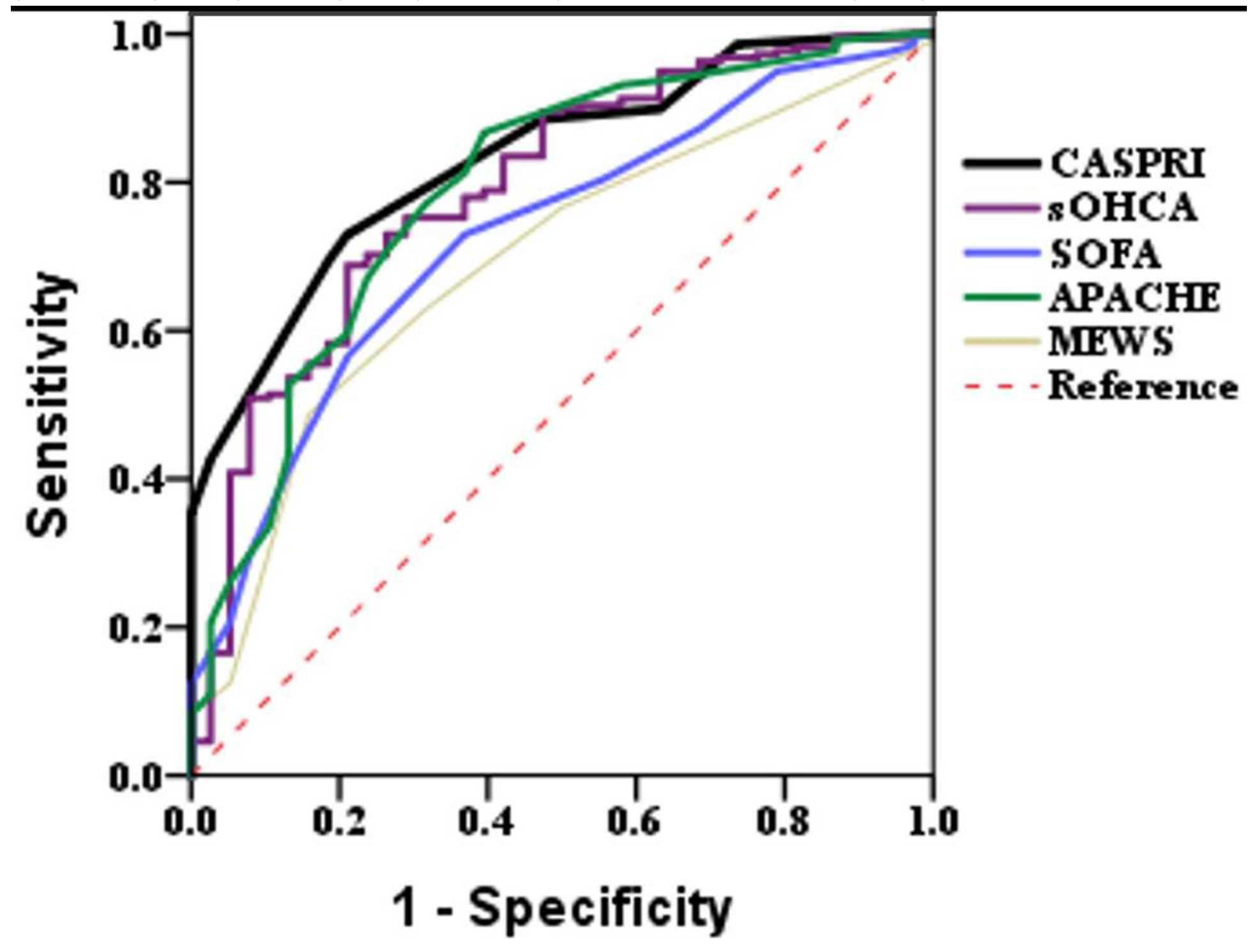

Figure 3

The efficacy of CASPRI score and other scores in evaluating the prognosis of ROSC patients. CASPRI score (AUC=0.896), sOHCA score (AUC=0.829), SOFA score (AUC= 0.815), APACHE II score (AUC=0.752), , and MEWS score (AUC=0.599). 


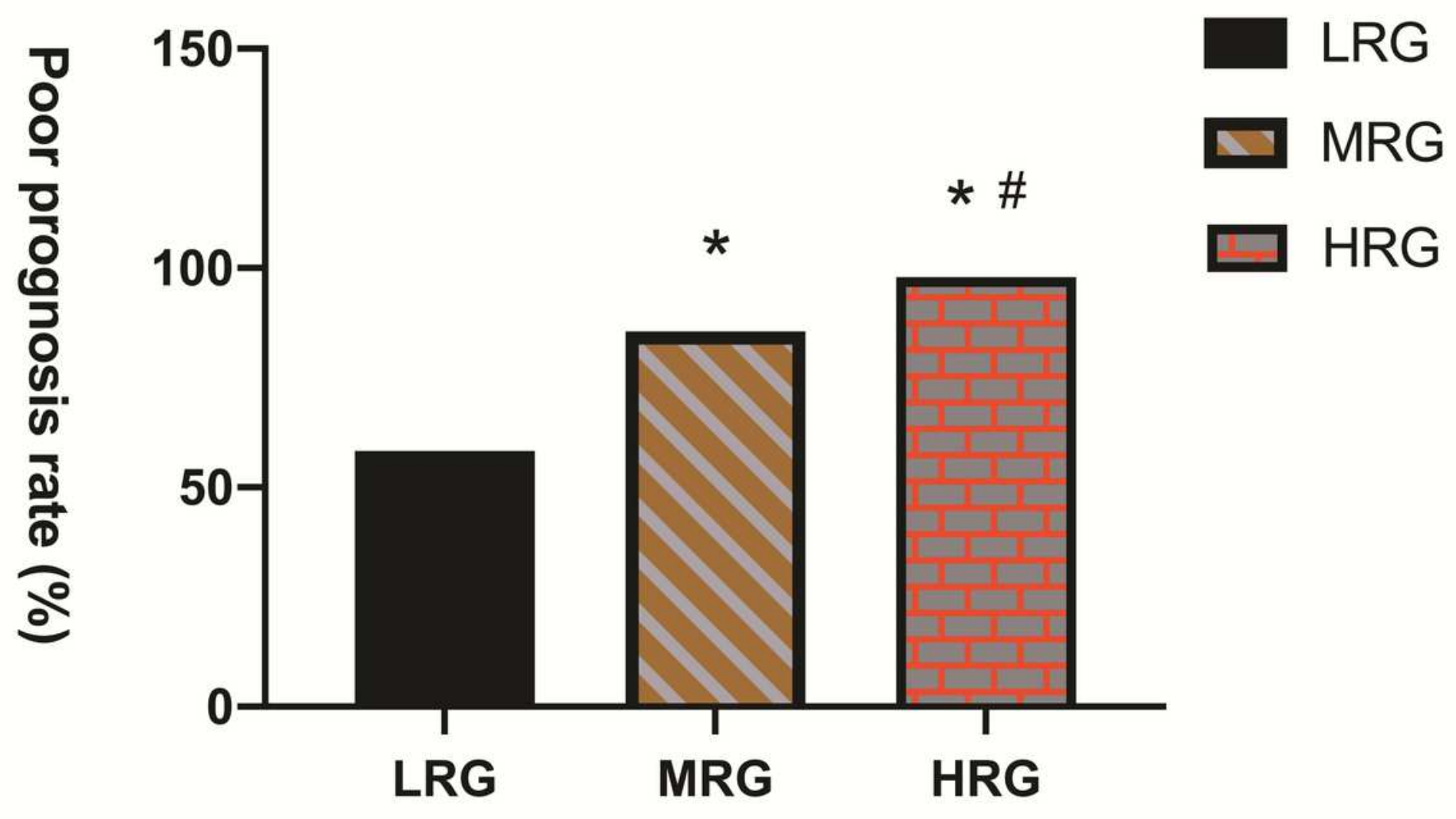

Figure 4

Comparison of poor neurological prognosis rate in CASPRI predicted high-, intermediate-, and low-risk groups. High-risk group (HRG), intermediate-risk group (MRG), and low-risk group (LRG). 


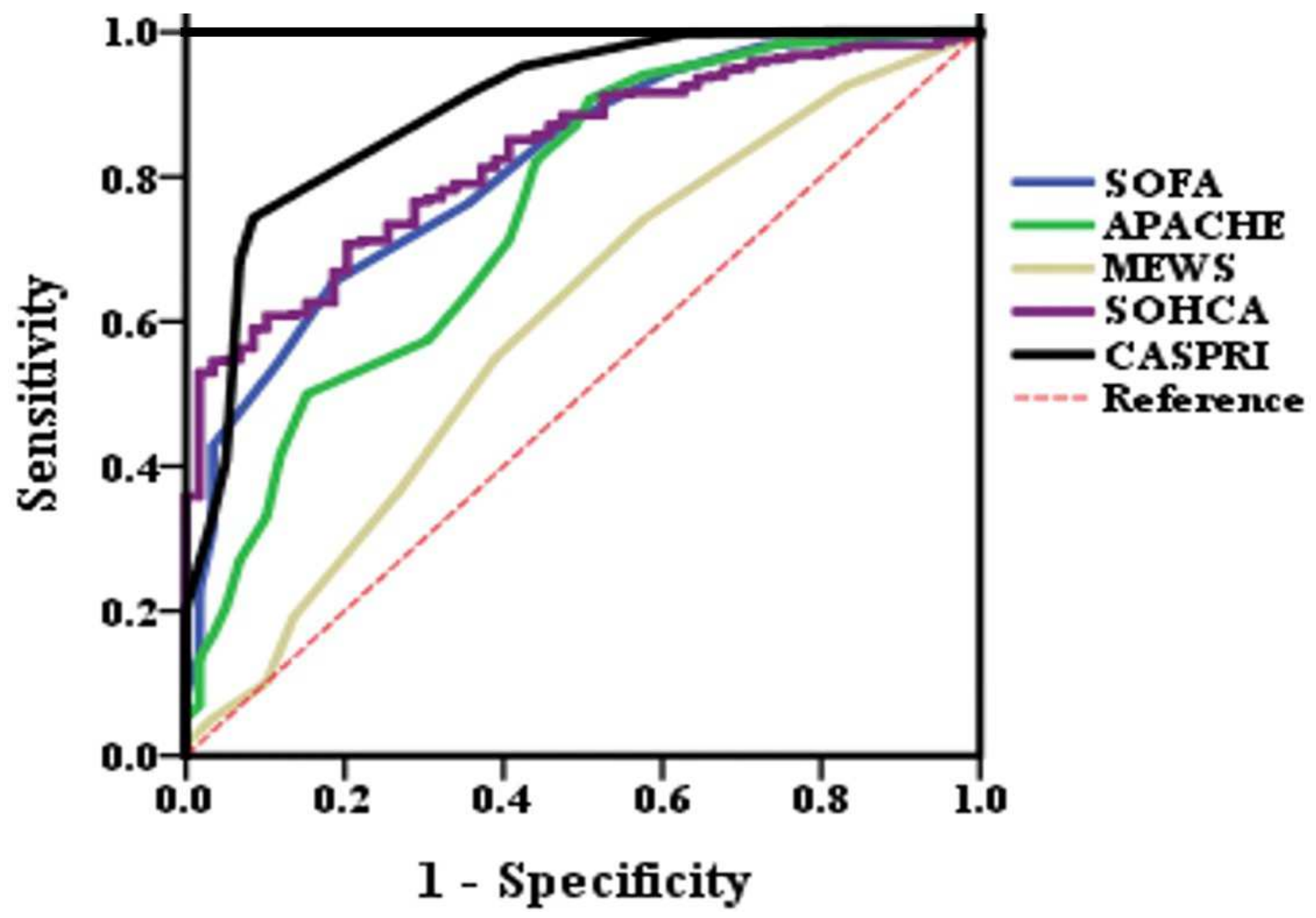

Figure 5

Verifying the efficacy of the CASPRI score in evaluating the neurological prognosis of ROSC patients. CASPRI score (AUC=0.831), SOFA score (AUC=0.726), APACHE II score (AUC=0.789), MEWS score (AUC=0.693), and sOHCA score (AUC=0.791).

\section{Supplementary Files}

This is a list of supplementary files associated with this preprint. Click to download.

- bmcarticle.bib

- bmcarticle.tex 